FHWA/IN/JTRP-2005/29

\title{
Impact of Camera and Lighting Position on Video Detection Precision
}

\author{
By \\ Avery Rhodes, EIT \\ Graduate Research Assistant \\ Kristofer Jennings, Ph.D \\ and \\ Darcy Bullock, Ph.D., PE \\ Professor of Civil Engineering
}
School of Civil Engineering
Purdue University
Joint Transportation Research Program
Project Number: C-36-17QQQ
File Number: 8-4-69
SPR-2869

\section{Prepared in Cooperation with the Indiana Department of Transportation and the U. S. Department of Transportation Federal Highway Administration}

The contents of this report reflect the views of the authors, who are responsible for the facts and the accuracy of the data presented herein. The contents do not necessarily reflect the official views and policies of the Indiana Department of Transportation or Federal Highway Administration at the time of publication. The report does not constitute a standard, specification or regulation.

\author{
Purdue University \\ West Lafayette, IN 47907 \\ May 2006
}




\section{TECHNICAL Summary}

INDOT Research

Technology Transfer and Project Implementation Information

TRB Subject Code: 54-5 Traffic Control Devices

Publication No.FHWA/IN/JTRP-2005/29, SPR-2869

May 2006

Final Report

\section{Impact of Camera and Lighting Position on Video Detection Precision}

\section{Introduction}

Video detection has become a popular replacement for traditional loop detectors at signalized intersections. While loop detectors are a relatively mature device, the experience with video detection is much more limited. The Indiana Department of Transportation suspended the deployment of video detection subsequent to a 2001 JTRP report detailing several problems with the technology. These included missed calls at night at intersections with limited lighting and the tendency for video detectors to extend detection zones significantly at night due to headlight reflection off of the pavement.

In 2002, suggestions to improve the performance of video detection were posed by video detection manufacturers. They were primarily concerned with

the placement of cameras, and suggested a preferred lateral offset and camera height.
In late 2003 and the summer of 2004, two test beds were constructed at signalized intersections in Noblesville, Indiana and West Lafayette, Indiana respectively. A camera was located at the vendor preferred location and several other cameras were located at slightly less optimal locations.

This report details the procedures used to evaluate three separate video detection systems at one of the test sites with respect to missed presence calls and false presence calls. A procedure to evaluate the consistency of detection zones between day and night lighting conditions is also presented. The conclusions of this report provide the Indiana Department of Transportation with important considerations when choosing detection technology at signalized intersections.

\section{Findings}

Autoscope (version 8.10), Peek UniTrak (version 2), and Iteris Vantage (Camera CAM-RZ3) were evaluated on the same traffic conditions at the Noblesville test site. All video detection systems were observed to fail to detect a large number of vehicles. Such performance is unacceptable and justifies INDOT's moratorium on video detection at signalized intersections. Furthermore, the high number of false calls is unacceptable due to the resulting motorist delay.

\section{Implementation}

This report provides a comprehensive evaluation of the Autoscope (version 8.10), Peek UniTrak (version 2), and Iteris Vantage (Camera CAMRZ3) stop bar video detection systems at signalized intersections. The deployment of video detections systems at signalized intersections is not recommended due to the following:
- Each video detection system showed a moderate to high number of missed and false calls over the two test periods.

- The loop detector showed only one missed call and 1 false call over both 48 hour test periods. The missed call was due to a wild vehicle path, while the false call was due to an unexplained eightsecond extension. 
- None of the three systems appeared to provide superior performance over the other three. The most accurate and reliable technology was the traditional loop detectors.
- The accuracy of all three systems appears to degrade with time and it appeared that a re-calibration was necessary only four months after the initial installation by factory representatives.

\section{Contacts}

For more information:

Prof. Darcy Bullock

Principal Investigator

School of Civil Engineering

Purdue University

West Lafayette IN 47907

Phone: (765) 494-2226

Fax: (765) 496-7996

E-mail: darcy@purdue.edu
Indiana Department of Transportation

Division of Research

1205 Montgomery Street

P.O. Box 2279

West Lafayette, IN 47906

Phone: (765) 463-1521

Fax: (765) 497-1665

\section{Purdue University}

Joint Transportation Research Program

School of Civil Engineering

West Lafayette, IN 47907-1284

Phone: (765) 494-9310

Fax: (765) 496-7996

E-mail: jtrp@ecn.purdue.edu

http://www.purdue.edu/jtrp 
This is a three-volume report; links to remaining two volumes are included in each volume.

TECHNICAL REPORT STANDARD TITLE PAGE



15. Supplementary Notes

Prepared in cooperation with the Indiana Department of Transportation and Federal Highway Administration.

\section{Abstract}

On well-illuminated approaches, vehicle headlight reflections on the pavement were observed to cause video detection units to activate early. This early activation results in a dramatic increase in the length of the effective vehicle detection zone. This observed variation in the effective length of the vehicle detection zone that varies by ambient lighting condition and camera placement presents a very serious impediment for traffic engineers to design vehicle extension intervals that operate correctly during day, night and transition periods. Furthermore, the stochastic variation in the length of the vehicle detection zone length has the potential to create driver expectancy issues. Tables are included that reports the observed average and range of detection zone length variations for 16 observed video cameras that were extensively calibrated by the manufacturer at the test site. The paper concludes by recommending near-side placement of video detection devices to reduce the stochastic variation in detection zone length.

17. Key Words

Traffic engineering, traffic signals, traffic surveillance, traffic management, traffic control devices, loops, intelligent transportation systems, statistical analysis, statistics, video detection, vehicle detector performance
18. Distribution Statement

No restrictions. This document is available to the public through the National Technical Information Service, Springfield, VA 22161

\begin{tabular}{|c|c|c|c|}
\hline $\begin{array}{c}\text { 19. Security Classif. (of this report) } \\
\text { Unclassified }\end{array}$ & $\begin{array}{c}\text { 20. Security Classif. (of this page) } \\
\text { Unclassified }\end{array}$ & 21. No. of Pages & 23 \\
\hline
\end{tabular}




\section{INTRODUCTION}

Video detection has become a popular replacement for traditional loop detectors at signalized intersections. While loop detectors are a relatively mature device, the experience with video detection is much more limited. The Indiana Department of Transportation suspended the deployment of video detection subsequent to a 2001 JTRP report detailing several problems with the technology. These included missed calls at night at intersections with limited lighting and the tendency for video detectors to extend detection zones significantly at night due to headlight reflection off of the pavement.

In 2002, suggestions to improve the performance of video detection were posed by video detection manufacturers. They were primarily concerned with the placement of cameras, and suggested a preferred lateral offset and camera height.

In late 2003 and the summer of 2004, two test beds were constructed at signalized intersections in Noblesville, Indiana and West Lafayette, Indiana respectively. A camera was located at the vendor preferred location and several other cameras were located at slightly less optimal locations.

This report details the procedures used to evaluate the consistency of detection zones between day and night lighting conditions. The conclusions of this report provide the Indiana Department of Transportation with important considerations when choosing detection technology at signalized intersections. 


\section{FINDINGS}

The video detection system analyzed in this research does not provide consistent detection zone sizes during day and night operation. This leads to unpredictable gap times and inefficient operation. 


\section{TABLE OF CONTENTS}

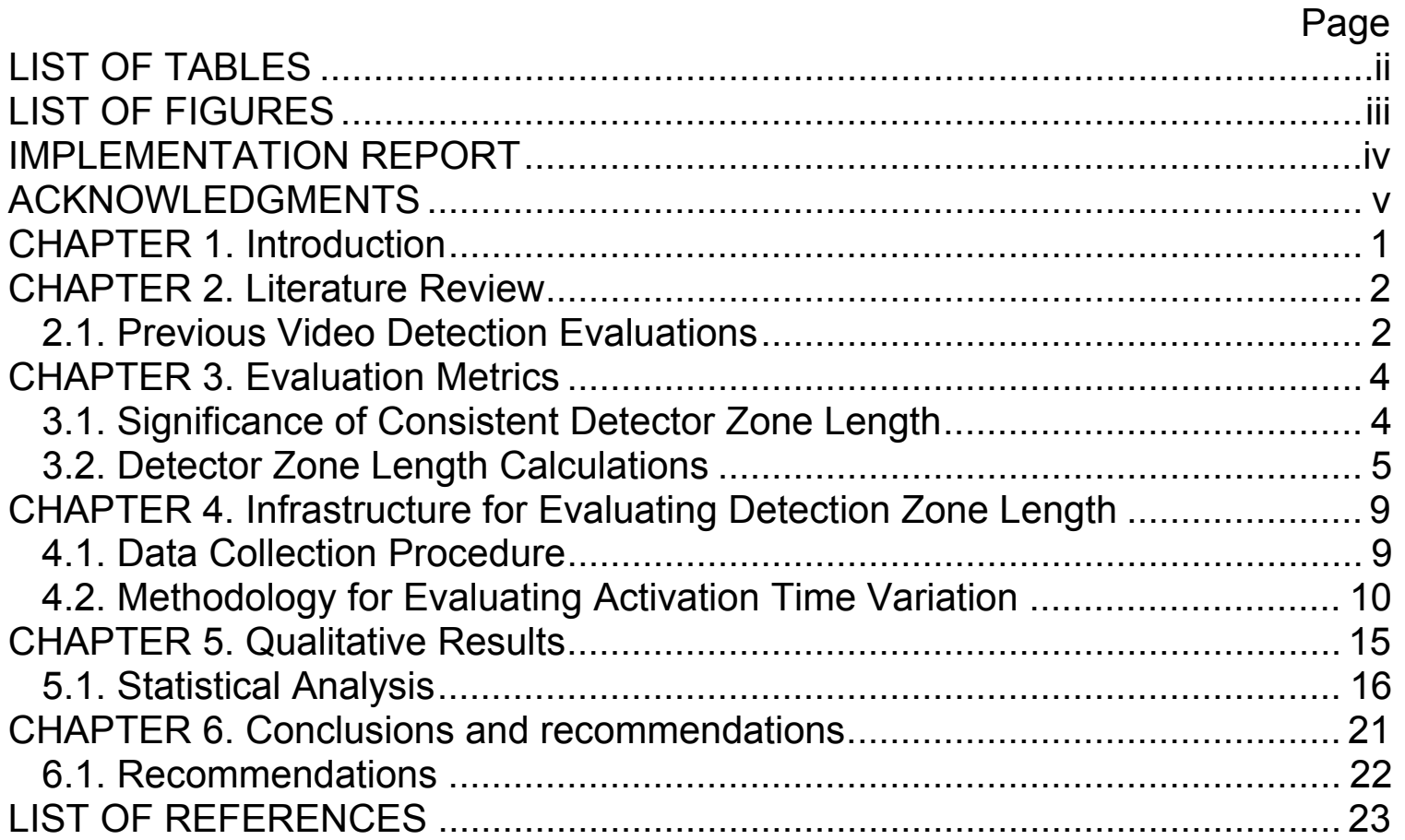




\section{LIST OF TABLES}

Table

Table 5-1: Statistical Analysis of Difference between Day and Night Activation Residuals............................................................................... 19 Table 5-2: Statistical Analysis of Difference between Day and Night De-activation

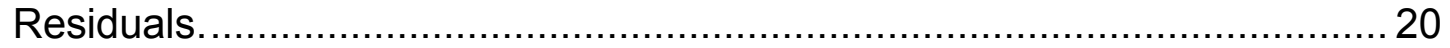




\section{LIST OF FIGURES}

Figure

Page

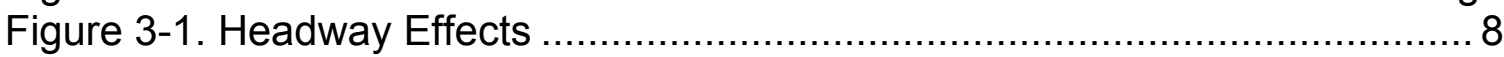

Figure 4-1. Example Camera Locations ............................................. 12

Figure 4-2. Illustration of Impact of Day versus Night Lighting on Detector Activation Precision.

Figure 4-3. Illustration of Impact of Video Detector Mounting Location on Detector



Figure 4-4. Example Histograms of Video Detection Timing Errors.................. 14

Figure 5-1. Video Detection Activation Residuals Histograms........................ 17

Figure 5-2. Video Detection Deactivation Residuals Histograms...................... 18 


\section{IMPLEMENTATION REPORT}

The use of video detection at signalized intersections is not recommended based upon the results reported in JTRP 2005/28. However, if used, the following operational characteristics of video detection have been identified that should be considered if video detection is installed at a location, including:

- The nearside above stop-bar video detection location should be used when possible to ensure the most consistent video detection zones function during both day and night operation.

- Even with this near side mounting of video detectors, traffic engineers should deploy video detection only when they are willing to accept the stochastic variation in detection zone performance that this paper has quantified at a well lit intersection configured by a highly trained manufacturer representative. Traffic engineers should also be cautioned that the stochastic variation in detection zones would be larger when used at poorly lit intersections.

- In the long term, it is essential that video detection manufacturers develop strategies to account for headlight effect in their detection algorithms to provide more consistent video detection zones across different lighting conditions. 
ACKNOWLEDGMENTS

This work was supported by the Joint Transportation Research Program administered by the Indiana Department of Transportation and Purdue University. The support for installing and configuring the video detection devices was provided by Dave Candey at Econolite Control Products. The contents of this paper reflect the views of the authors, who are responsible for the facts and the accuracy of the data presented herein, and do not necessarily reflect the official views or policies of the Federal Highway Administration and the Indiana Department of Transportation, nor do the contents constitute a standard, specification, or regulation. 


\title{
CHAPTER 1. INTRODUCTION
}

\begin{abstract}
Vehicle detection using video cameras has become an increasingly popular alternative to loop detectors at signalized intersections. Although many product evaluations have been completed, the majority of research has focused on freeway applications or used metrics such as speed and volume that may not represent the performance of a device for presence or passage detection at a signalized intersection. Subsequent research evaluated the performance of video detectors for presence detection at signalized intersections $(1,2)$. This research quantifies the variation in detector zone lengths between day and night operation at a well illuminated intersections using video detection devices that were extensively calibrated by the vendor.
\end{abstract}




\section{CHAPTER 2. LITERATURE REVIEW}

\subsection{Previous Video Detection Evaluations}

Grenard et al. (2) proposed an evaluation from a presence detection perspective. A real time test was completed with data collected from both a video detector and a loop detector. The periods during the test where the two detectors were not in agreement were observed on a recorded video to determine the cause of the discrepancy. The advantage of this procedure is that the detector is operating in a real world situation, and not from a pre-recorded video that may cause poorer performance due to poorer video quality or because the video detection system is not operating with the camera as it normally does in the field where the system can potentially adjust camera settings in real time to improve performance.

MacCarley and Palen (3) have suggested a procedure, with video detection in mind, that considers the impacts of detector missed calls and false calls. Specifically, during the green phase, the phase can either be incorrectly extended due to false calls or incorrectly terminated early due to missed calls. Similarly during a red phase, a false call can be placed to incorrectly call a phase or a missed call may prevent a phase from being called.

Bonneson (4) accounted for the impact of video detection errors to signal operations through the metrics of discrepant call frequency and error rate. Discrepant call frequency is the number of discrepant calls per signal cycle and error rate is the ratio of error calls to correct calls. This research also identified two metrics that would be easy to collect and could be related to intersection performance. These metrics are phase max-out and control delay. Using these two metrics, different detector set-ups for the video detectors were evaluated. 
These metrics could also be used for a comparison of a video detector to a loop detector. Phase max-out refers to the percentage of signal phases that terminate because they reach the max green time rather than gapping out. Theoretically, an efficiently operating intersection will gap out more frequently than it reaches the max green time. Obviously, at an oversaturated intersection, the phases would tend to reach the max green time and it would be difficult to distinguish between the performances of multiple detectors. The second metric, control delay, simply refers to the amount of delay that is incurred by the motorists due to the signal control.

Baculinao (5) documents a specific situation in the City of Santa Clarita, California where the use of video detection mandated the modification of signal timings due to "downlane occlusion". Downlane occlusion occurs when a large vehicle, such as a semi-truck and trailer, occlude vehicles behind it because of its large height. In this specific case, the author was concerned that the signal controller would incorrectly calculate 'added initial green' times because of the missed vehicles by the video detection. 


\section{CHAPTER 3. EVALUATION METRICS}

\subsection{Significance of Consistent Detector Zone Length}

This paper introduces a new metric for the evaluation of detectors at signalized intersections. This metric can be viewed as a measure of the consistency of detection zones. To provide predictable and consistent data to the traffic signal controller, a detector must provide consistent detector zones across varying traffic and environmental conditions.

From a traffic engineering perspective, there are several reasons that a crisp and consistent detection zone is desirable. The traffic signal controller reacts to traffic conditions through the information that is gathered from vehicle detectors. When a detector operates differently, due to a factor such as the ambient lighting condition, the traffic signal will effectively operate differently as it responds to the inconsistent information from the vehicle detector. The traffic engineer will therefore have to program not only by time of day but also by day of year to ensure consistent operation across lighting conditions. The traffic engineer may not be able to account for other factors that effect detector performance but do not occur on a predictable schedule.

Accurate detection is not only noticeable from the traffic engineer's perspective but also from the general public's perspective as highlighted in a recent Washington Post article describing the problems the Virginia Department of Transportation encountered with a new video detection system and the corresponding frustration experienced by motorists due to the inefficient transportation system (6).

The theoretical foundation for determining when to terminate a green phase is based upon the flow-density curve to determine when the flow rate 
drops below a predetermined threshold (Figure 3-1a). For example, if vehicle headways are consistently in the range of $2.0 \mathrm{~s}$, the approach can be assumed to still be in the saturated regime. However, when headways fall below a prescribed threshold, for example, Point 2 in Figure 3-1a, it is often appropriate to terminate the phase and service another phase with a standing queue. Even very small changes in the measurement of headway will have a profound effect on the measurement of flow rate as shown on the right side of Figure 3-1a. A measured headway of 2.0 seconds corresponds to a flow rate of 1800 vehicles per hour (VPH). Changing the measured headway only slightly to 3.0 seconds results in a corresponding flow rate of only $1200 \mathrm{VPH}$. Similarly, as the headway changes to 4.0 seconds, the corresponding flow rate drops to $900 \mathrm{VPH}$.

Most signalized intersection detectors do not directly measure headway; instead they measure gaps in the traffic stream (Figure 3-1b). When inaccurate gap measurements are observed, such as when a vehicle's headlights activate a video detector early, the controller may extend the green signal phase unnecessarily because the gap times appear smaller than they actually are.

Consequently, one can conclude that crisp detection zone operation is essential during the green phase. During red signal phases where the signal reacts to the presence of a vehicle and not to a measured value such as headway, a crisp, deterministic detector activation time is less crucial to efficient signal operations. Quantifying the consistency of the activation and de-activation of a detection zone is discussed in the subsequent sections.

\subsection{Detector Zone Length Calculations}

The headlight effect on gap measurement is illustrated in Figure 3-1c. Dimensions illustrated on the figure are categorized into three measurement units (distance, velocity, and time) and divided by dashed lines.

The basic Greenshield models of traffic flow are shown in Figure 3-1a \& b. The relationship between flow and density is shown in Figure 3-1a. As the 
density of traffic increases, the flow rate also increases up until point 1 where jam density is reached.

In Figure 3-1b the relationship between flow and headway is displayed. As headway increases, the corresponding flow rate decreases. This is important from a traffic signal operations perspective because headway is often used to measure flow rate to determine when to extend a green phase for heavy traffic flow or terminate a green phase once the flow rate of traffic drops below a certain threshold.

Headway is indirectly used at signalized intersections by measuring the gap between vehicles. The gap is measured by the time that a detector is off between vehicle actuations.

At night, video detectors may activate early due to headlight reflection off of the pavement. This early activation will cause the gap times and indirectly headway to be measured inaccurately. As headlights activate video detectors early, the effective length of the vehicle is larger than the actual length of the vehicle and the controller may extend phases unnecessarily due to the measured headways being shorter than the actual headways.

In Figure 3-1c, the headlight effect and its impact on gap measurement is illustrated. The actual length of the vehicle is represented by $L_{c}$ while the effective length of the vehicle is shown by $L_{e}$. The effective vehicle length is longer because the headlight reflection is being detected as a vehicle presence.

The headway $\left(\mathrm{H}_{\mathrm{a}}\right)$ is a function of the actual gap and the speed and length of the vehicle as shown in Equation 1.

$$
H_{a}=G_{a}+\frac{L_{c}}{V_{c}}
$$

The actual gap is represented by $G_{a}$, however the actual gap is sensed as $G_{s}$, because of the headlight effect which reduces the gap time by $G_{h}$ as shown by Equation 2.

$$
G_{a}=G_{s}+G_{h}
$$


The final actual headway $\left(\mathrm{H}_{\mathrm{a}}\right)$ is a function of the sensed gap, plus the gap time reduced by the headlight effect and the speed and length of the vehicle as shown in Equation 3.

$$
H_{a}=G_{s}+G_{h}+\frac{L_{c}}{V_{c}}
$$

However, the sensed headway $\left(H_{s}\right)$ is reduced by the headlight effect $\left(G_{h}\right)$ and is calculated by Equation 4.

$$
H_{s}=G_{s}+\frac{L_{c}}{V_{c}}
$$

The measured gap times directly affect the measured headway. The measured headway is $\mathrm{H}_{s}$, which is reduced by $\mathrm{H}_{h}$ due to headlights. The actual headway is the sum of $\mathrm{H}_{s}$ and $\mathrm{H}_{h}$, as represented by $\mathrm{H}_{\mathrm{a}}$. 


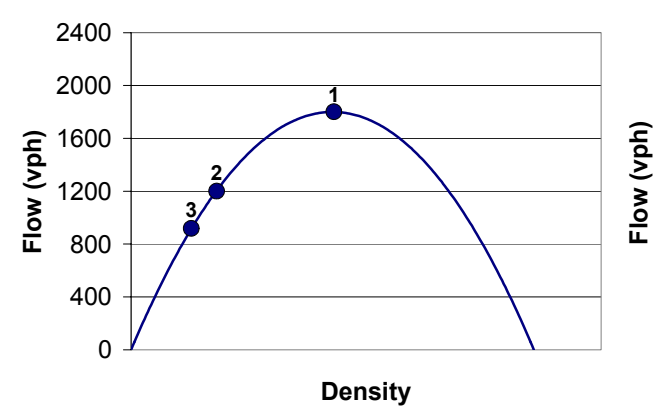

a) Relationship between Density and Flow

\section{Measurement}

Units

Distance

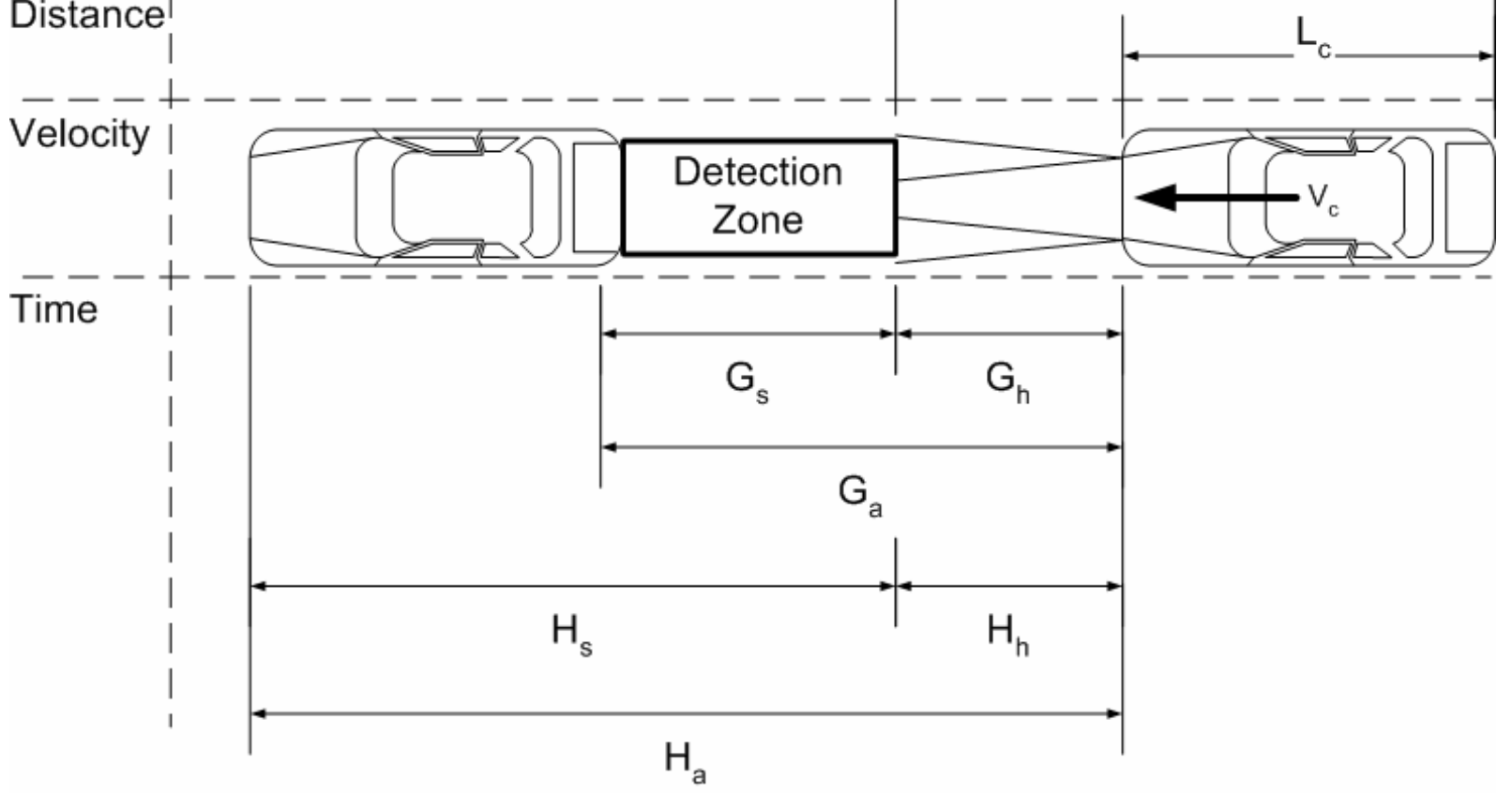

c) Illustration of Relationship between Headway and Gaps b) Relationship between Headway and Flow

Figure 3-1. Headway Effects 


\section{CHAPTER 4. INFRASTRUCTURE FOR EVALUATING DETECTION ZONE LENGTH}

A test site was constructed in West Lafayette, Indiana to serve as a test bed for video detection technology. Each approach was instrumented with inductive loop detectors and video detectors. The loop detectors provide consistent performance to which the performance of the video detectors can be compared.

There were four camera positions per approach at this location, for a total of sixteen cameras. The four camera positions for the Northbound approach are shown in Figure 4-1, and are representative of all approaches. Camera 1 is installed at the vendor recommended location of 40 feet high and laterally positioned inline with the lane line between the left-turn lane and the leftmost through lane. Camera 2 is located at a slightly less optimal lateral position at the top of the signal pole. Camera 3 is located at the recommended lateral position but it is positioned on the signal mast arm at a height of only 25 feet. Finally, Camera 4 is positioned above the detected approach looking directly down into the detection area.

\subsection{Data Collection Procedure}

Phase states and detector states were logged to a text file during the test period for later use during data reduction. Additionally, a digital video of each approach was captured with a screen overlay giving the status of the phases and detectors in real time. Example overlays are shown in Figure 4-2 and Figure 4-3. The overlays indicate loop detector and video detector states with labels that either indicates an active detector (dark black legible text) or an inactive detector 
(white hollow text). The states for the through lane detectors are shown on the right side of the video directly in front of the through lanes and the state of the left-turn lane detectors are shown on the left-side of the video in front of the leftturn lane. The phase states are also represented for the through lanes and leftturn lanes by a standard 3-head signal face.

In Figure 4-2 an example of the difference in performance between day and night on a single camera is shown. Each of the cameras is configured so that the detection zone matches the loop detection zone as closely as possible. All of the examples refer to the Northbound through phase (Ф2).

In Figure 4-2a each of the through lane video detectors is activating at nearly the same moment as the through lane loop detector, in fact in this example Camera 2 activates at the exact same time as the loop detector. In Figure $4-2 b$, the activation times of the camera are significantly shifted towards earlier activation by more than 1.1 seconds due to headlight reflection coming into the video detection zone prior to the vehicle arriving.

In Figure 4-3 an example of the difference in performance due to camera positioning is displayed. In Figure 4-3a, the far side mounted cameras activate 1 to 3 seconds earlier than the loop detector in the through lanes due to the headlight effect. However, in Figure 4-3b the above mounted near side camera activates at nearly the same moment as the loop detector, with a difference in activation time of only 0.134 seconds for the through lanes.

\subsection{Methodology for Evaluating Activation Time Variation}

An example of an activation time histogram and deactivation time histogram are shown in Figure 4-4. These examples are merely used to illustrate the concept of the histograms while actual results from the test are presented later in the paper.

An example histogram of the activation time residuals is shown in Figure 4-4a. A thin vertical black line represents the loop activation time at 0.0. The relative activation time for the video detector is represented along the horizontal 
axis by the number of seconds prior to loop activation (to the left of 0.0 ) or by the number of seconds subsequent to loop activation (to the right of 0.0 ). On the vertical axis, the frequency of each video detector activation time is represented. A similar configuration is shown for a deactivation histogram in Figure 4-4b.

In the first example of activation times (Figure 4-4a), the front of Vehicle A activates the loop detector at $0.0 \mathrm{~s}$. The video detector activates at 0.3 seconds after the loop detector. A more common night occurrence is the video detection activating prior to the loop detector, as is the case for Vehicle B. In this example, the loop detector again activates at $0.0 \mathrm{~s}$, but the video detector activated approximately 1.3 seconds early.

Examples for deactivation times are shown in Figure 4-4b. In this figure, the back of Vehicle A leaves the detection zone and deactivates the loop at 0.0s, however the video detector is slower to deactivate and does so 1.3 seconds after the loop. The video detector deactivates more quickly in the example for Vehicle $B$, at 0.3 seconds after the back of the vehicle leaves the loop. 


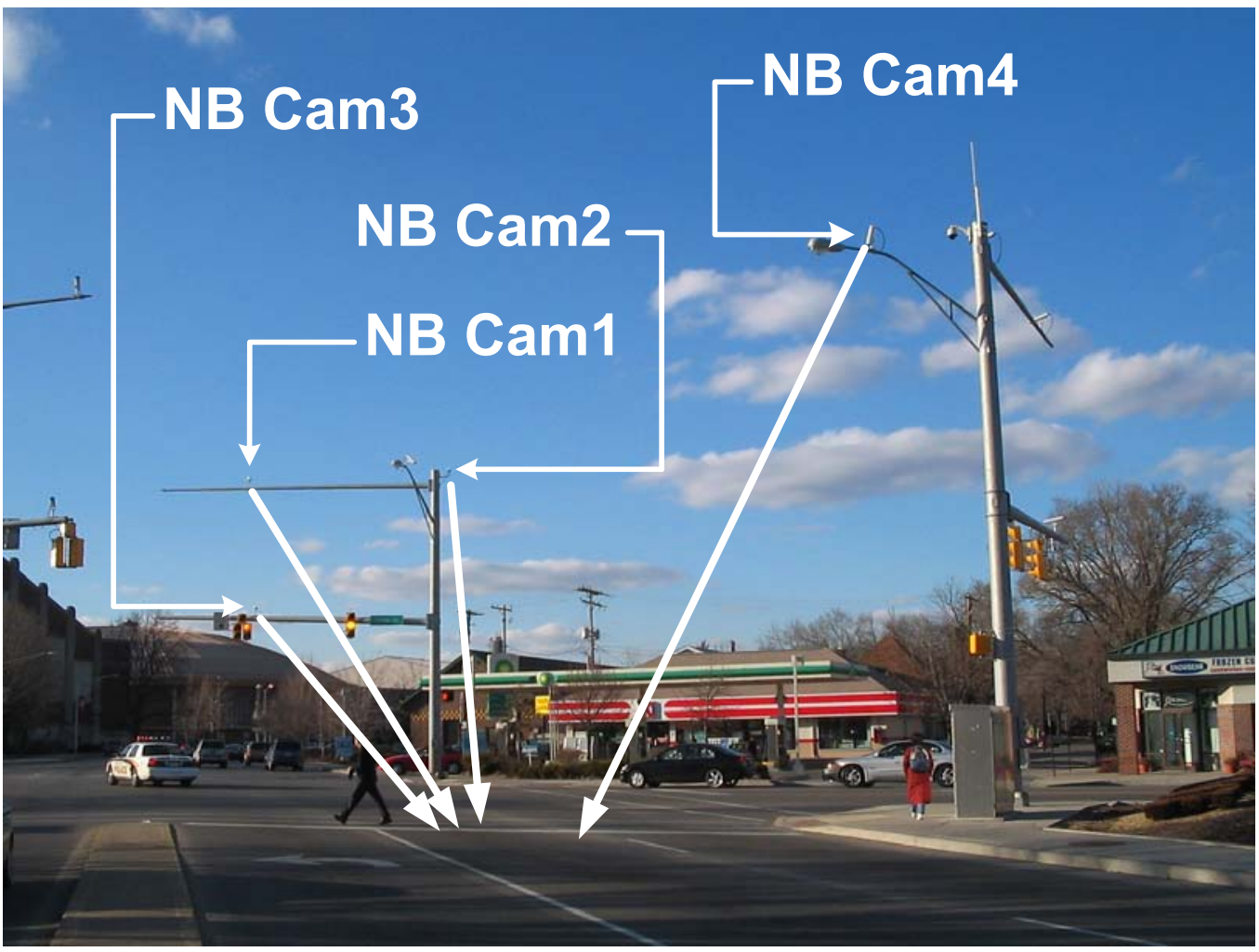

Northbound Approach - West Lafayette, Indiana

Figure 4-1. Example Camera Locations 


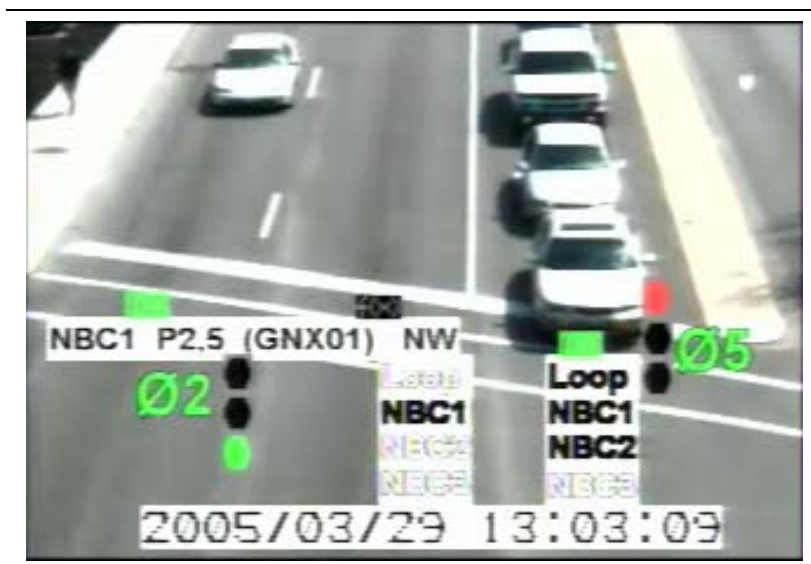

a) Day Time Camera Activations

Cam1: -0.134 seconds

Cam2: 0.000 seconds

Cam3: -0.100 seconds

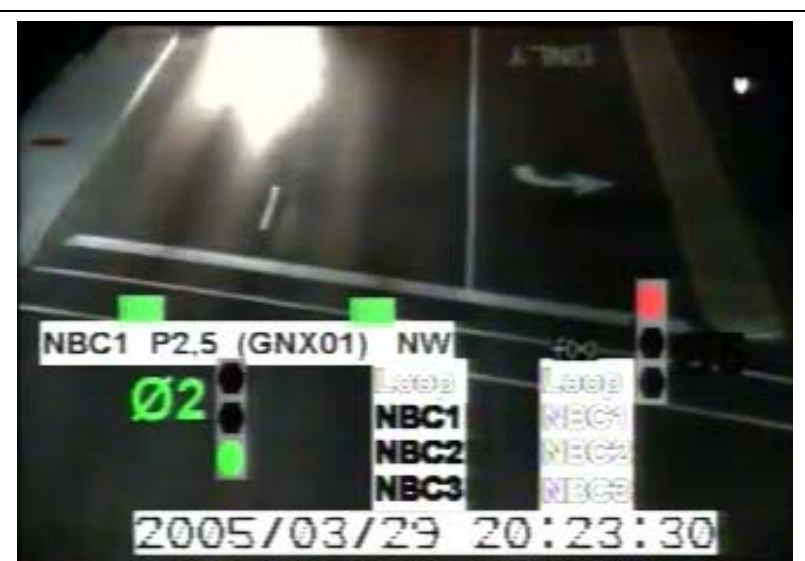

b) Night Time Camera Activations

Cam1: -1.335 seconds Cam2: -1.101 seconds

Cam3: -1.502 seconds

Figure 4-2. Illustration of Impact of Day versus Night Lighting on Detector Activation Precision.

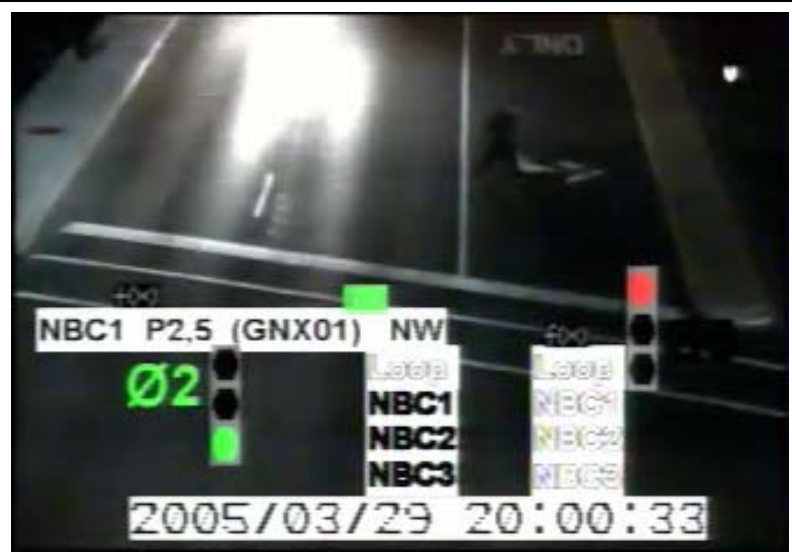

a) Far Side Camera Activations

Cam1: -1.468 seconds

Cam2: -1.702 seconds

Cam3: -3.003 seconds

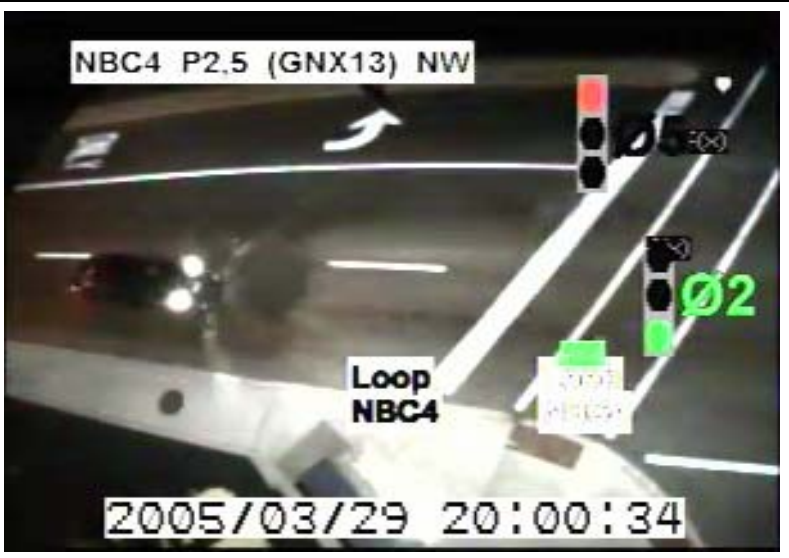

b) Above Stop Bar Camera Activation Cam 4: +0.134 seconds

Figure 4-3. Illustration of Impact of Video Detector Mounting Location on Detector Activation Precision. 
Time Difference between Loop Detector and Video Detector Activation (Seconds)

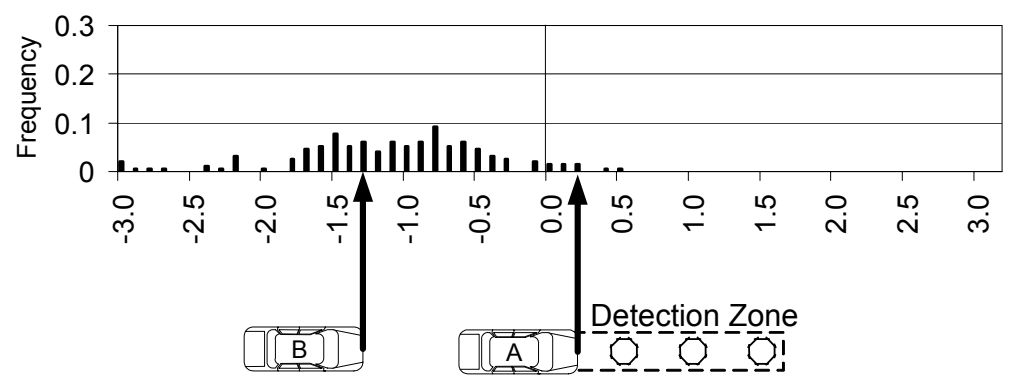

a) Example Activation Histogram

Time Difference between Loop Detector and Video Detector Deactivation (Seconds)

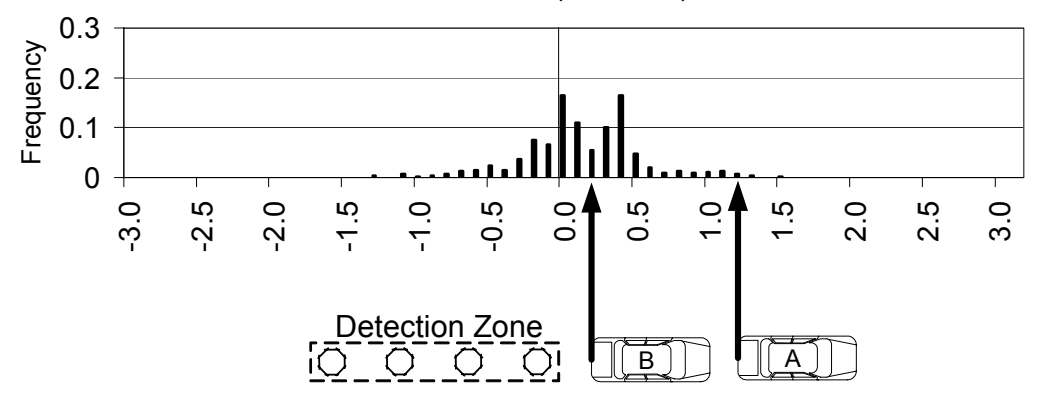

b) Example Deactivation Histogram

Figure 4-4. Example Histograms of Video Detection Timing Errors 


\section{CHAPTER 5. QUALITATIVE RESULTS}

The variations in distributions of activation and deactivation times are shown in histograms in Figure 5-1 and Figure 5-2. The thin black line at the 0.0 second mark represents the point at which the loop detector activates or deactivates. The distributions of the activation and deactivation times of the video detectors are relative to this zero point. The histograms display both a distribution from a day period and a night period. The data was collected on January $25^{\text {th }}$, 2005, with the day period covering the hours between 12:00 PM and 4:00 PM, and the night period covering the hours between 8:00 PM and 12:00 AM. The data collection occurred on the Northbound through phase (\$2).

The weather conditions during the test were clear. Snow had fallen at the site on January $20^{\text {th }}, 2005$, however the snow on the roadway had already melted by the test date. It should be expected that the headlight effect would be even more pronounced when the pavement is wet from rain or melting snow, therefore, a day without these conditions was chosen for the test.

Qualitatively looking at the activation histograms displayed in Figure 5-1, it is immediately apparent that the night and day distributions differ considerably for cameras 1 through 3 (Figure 5-1a, b \& c). The distribution of activation times for camera 4 (Figure 5-1d) is much more consistent between day and night periods.

The activation histograms show a consistent shift towards earlier activation at night as compared to the day time periods. Even on camera 4 (Figure 5-1d) there is a slight shift towards earlier activation during the night time periods, but it is far less dramatic than the shift on the first three camera positions (Figure 5-1a, b \& c). 
The deactivation histograms shown in Figure 5-2 are much more consistent across the day and night periods. Observing all four histograms (Figure 5-2a, b, c, \& d) there is no discernible shift for any of the camera positions.

In order to verify the qualitative observations, statistical analysis was performed for each camera to quantify the differences between day and night on all of the activation and deactivation histograms for all four directional approaches at the West Lafayette test intersection.

\subsection{Statistical Analysis}

The student's t-test is used to verify statistically that there exists a difference in the means of the on-times and off times between the day and night periods. This test is summarized in Table 5-1 and Table 5-2 for the activation and deactivation residuals, respectively. The figures include the mean, standard deviation, sample sizes for both day and night, as well as the t statistic for the comparison of the two means and the resulting statistical conclusion of significance $(\alpha=0.05)$. The $95 \%$ confidence interval is also included for the activation and deactivation times in these two tables.

For activation times, all of the cameras in the test with the exception of SB2 showed a statistically significant earlier activation time for the night period when compared to the day period. This result was expected, as by observation, the video detectors tend to activate early because of headlights. As can be seen in the difference in means column of Table 5-1, the Camera 4 position demonstrated the lowest average difference in means on all approaches. This camera position is mounted directly over the detection zone and is therefore less susceptible to early activation from headlight reflection.

The deactivation times were more consistent between the day and night periods although 9 of 16 cameras still showed a statistically different deactivation time between day and night. The average deactivation times were not 
consistently earlier or later than the average times during the day. Also the differences in average deactivation times were not as pronounced as they were for the average activation times. Therefore, while there is inconsistent performance in the deactivation times it is relatively minor and does not seem directly correlated to an observable phenomenon such as headlight effect.

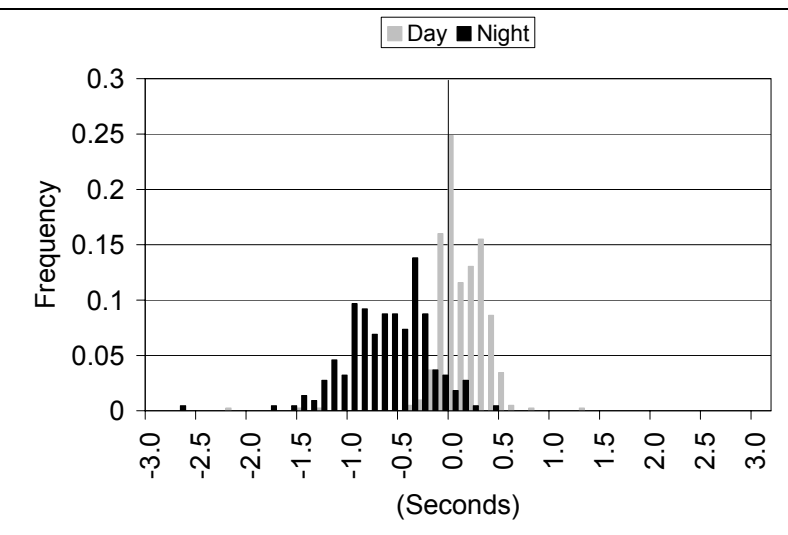

a) Camera 1

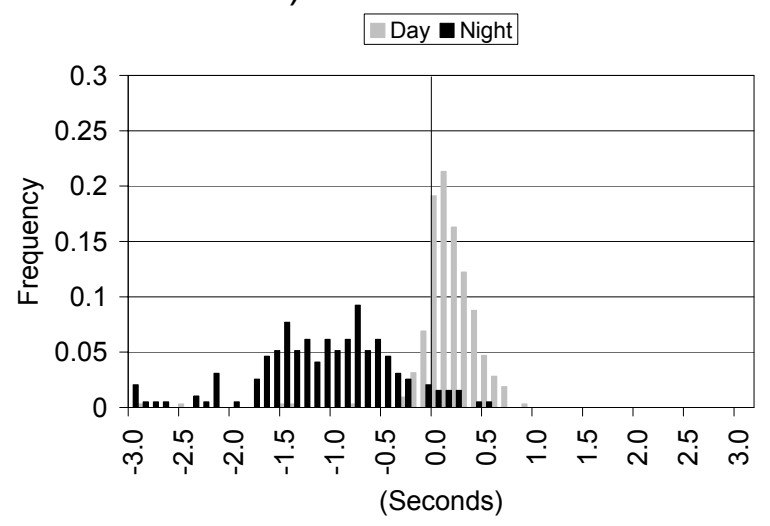

c) Camera 3

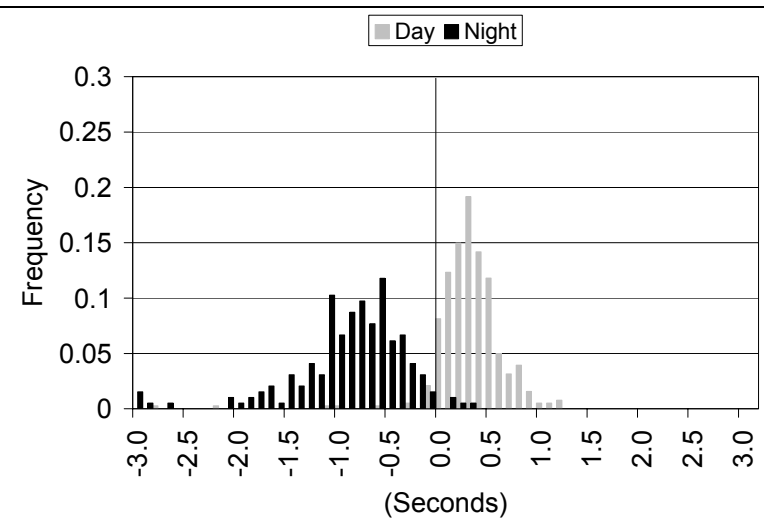

b) Camera 2

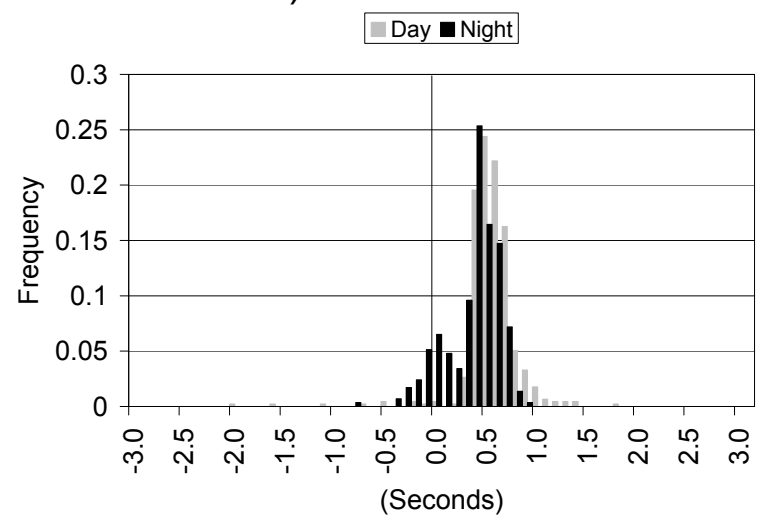

d) Camera 4

Figure 5-1. Video Detection Activation Residuals Histograms. 


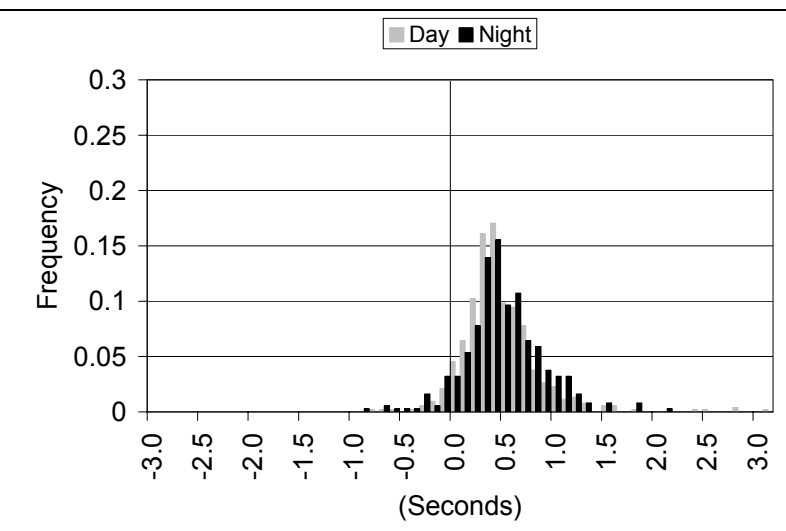

a) Camera 1

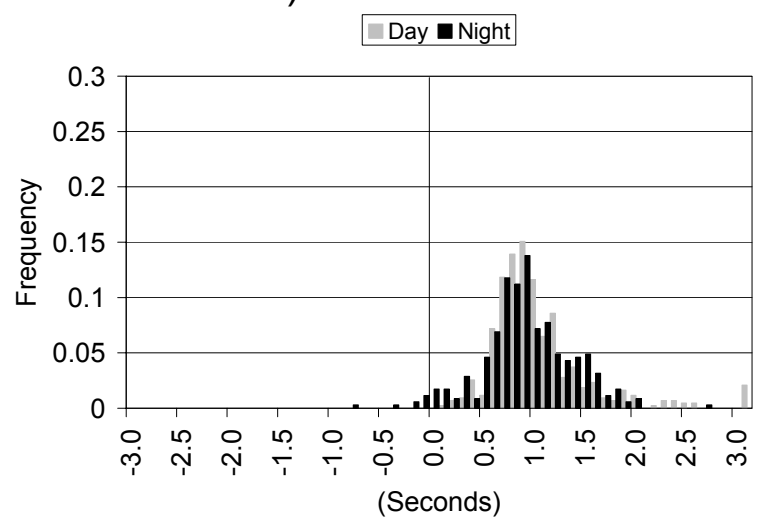

c) Camera 3



b) Camera 2

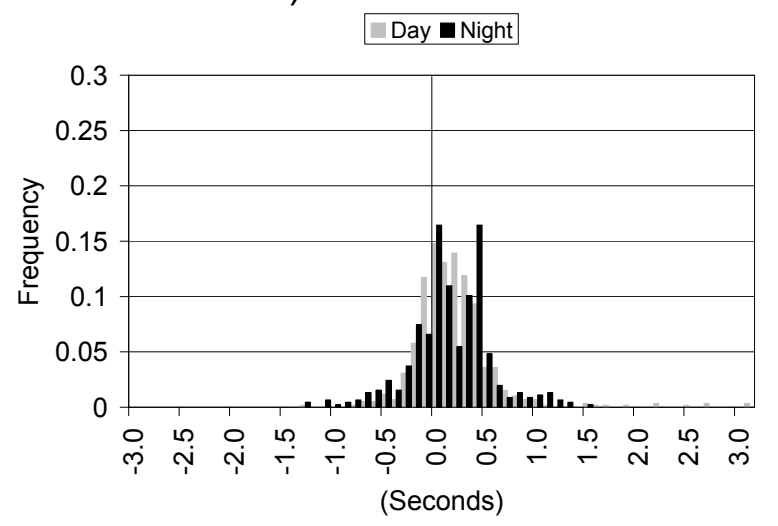

d) Camera 4

Figure 5-2. Video Detection Deactivation Residuals Histograms. 
Table 5-1: Statistical Analysis of Difference between Day and Night Activation Residuals.

\begin{tabular}{|c|c|c|c|c|c|c|c|c|c|c|c|c|c|}
\hline \multirow{4}{*}{$\begin{array}{l}\text { Cam. } \\
\text { NB1 }\end{array}$} & \multirow{2}{*}{\multicolumn{3}{|c|}{$\begin{array}{c}\text { Day } \\
\text { Activation } \\
\text { Residual } \\
\mathrm{t}_{\mathrm{v}, \text { on }}-\mathrm{t}_{\mathrm{l}, \mathrm{on}} \\
\text { (seconds) }\end{array}$}} & \multirow{2}{*}{\multicolumn{3}{|c|}{$\begin{array}{c}\text { Night } \\
\text { Activation } \\
\text { Residual } \\
\mathrm{t}_{\mathrm{v}, \mathrm{on}}-\mathrm{t}_{\mathrm{l}, \mathrm{on}} \\
\text { (seconds) }\end{array}$}} & \multicolumn{7}{|c|}{$\begin{array}{l}\text { Statistical Comparison Between Loop } \\
\text { And Video Detection Activation Time } \\
\text { (day vs. night) }\end{array}$} \\
\hline & & & & & & & \multirow{2}{*}{ 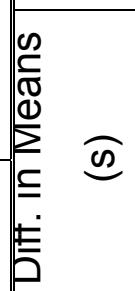 } & \multirow{2}{*}{ 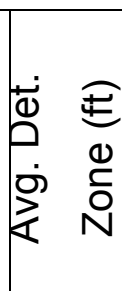 } & \multirow{2}{*}{ 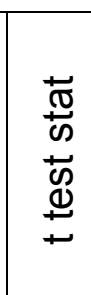 } & \multicolumn{4}{|c|}{$\begin{array}{c}\text { Statistical Results } \\
(\alpha=0.05)\end{array}$} \\
\hline & $\mathrm{vg}$. & $\begin{array}{l}\text { Std. } \\
\text { Dev }\end{array}$ & $n$ & & $\begin{array}{l}\text { Std. } \\
\text { Dev }\end{array}$ & $\mathrm{n}$ & & & & & iff & & \\
\hline & 1 & $0 ?$ & 405 & -0.7 & 0.4 & 217 & 0.76 & 39.1 & & & $\checkmark$ & 0.75 & 35 \\
\hline NB2 & 0.3 & & & & 0.6 & 194 & 1.18 & 60. & & & 7 & & .28 \\
\hline$\overline{N R}$ & 0. & & $a_{1}$ & & 07 & 194 & .22 & 62.8 & & & & & .29 \\
\hline & & & & & & 292 & 0 & 10.3 & & & & & 0.24 \\
\hline SB1 & & & & & 0.4 & 01 & 99 & 50.9 & & & & & .07 \\
\hline $\mathrm{SE}$ & & & & & 0.5 & & 4 & -7.2 & & & & & 0.42 \\
\hline SB3 & & & & & & & & 518 & & & & & 08 \\
\hline SB4 & & & & & 0.2 & 119 & 3 & 0.1 & & & & ? & 24 \\
\hline EB1 & & & & & & 287 & 0 & & & & 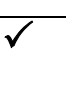 & & .46 \\
\hline $5 \square$ & & & & & & 252 & 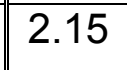 & & & & 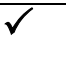 & & 220 \\
\hline EB3 & & 6 & 288 & & 1.1 & 4 & 02 & 9.1 & & & & & .13 \\
\hline EB4 & & & & & & 345 & 38 & 38.8 & & & $\checkmark$ & & .95 \\
\hline WB1 & 0.0 & 0.5 & & -0.0 & 0.1 & III & 40 & 01.1 & & & $\checkmark$ & 1.21 & 53 \\
\hline WB2 & 2.1 & 1 & 2 & & 0.6 & & 39 & 61 & & & 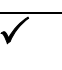 & 0.8 & 1.78 \\
\hline WB & 0.7 & 0.4 & 68 & 3 & 0.7 & 93 & 6 & 46.7 & & & $\checkmark$ & & 1.14 \\
\hline$\sqrt{2}$ & 0.5 & .1 & 136 & & . & 124 & 0.77 & 34.0 & & & $\checkmark$ & 0.68 & 0.92 \\
\hline
\end{tabular}


Table 5-2: Statistical Analysis of Difference between Day and Night De-activation Residuals.

\begin{tabular}{|c|c|c|c|c|c|c|c|c|c|c|c|}
\hline \multirow{4}{*}{$\begin{array}{l}\text { Cam. } \\
\text { NB1 }\end{array}$} & \multirow{2}{*}{\multicolumn{3}{|c|}{$\begin{array}{c}\text { Day } \\
\text { Activation } \\
\text { Residual } \\
t_{\mathrm{v}, \text { on }}-\mathrm{t}_{\mathrm{l}, \mathrm{on}} \\
\text { (seconds) }\end{array}$}} & \multirow{2}{*}{\multicolumn{3}{|c|}{$\begin{array}{c}\text { Night } \\
\text { Activation } \\
\text { Residual } \\
\mathrm{t}_{\mathrm{v}, \text { on }} \mathrm{t}_{\mathrm{l}, \mathrm{on}} \\
\text { (seconds) }\end{array}$}} & \multicolumn{5}{|c|}{$\begin{array}{l}\text { Statistical Comparison Between Loop } \\
\text { And Video Detection Activation Time } \\
\text { (day vs. night) }\end{array}$} \\
\hline & & & & & & & \multirow{2}{*}{ 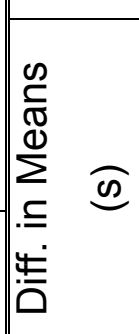 } & \multirow{2}{*}{ 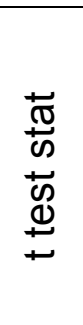 } & \multicolumn{3}{|c|}{$\begin{array}{l}\text { Statistical Results } \\
\qquad(\alpha=0.05)\end{array}$} \\
\hline & \multirow{2}{*}{\begin{tabular}{|c|} 
Avg. \\
0.4 \\
\end{tabular}} & \multirow{2}{*}{$\begin{array}{c}\text { Std. } \\
\text { Dev }\end{array}$} & \multirow{2}{*}{$\begin{array}{c}n \\
527\end{array}$} & \multirow{2}{*}{$\begin{array}{c}\text { Avg. } \\
0.4\end{array}$} & \multirow{2}{*}{$\begin{array}{c}\text { Std. } \\
\text { Dev }\end{array}$} & \multirow{2}{*}{$\begin{array}{c}n \\
373\end{array}$} & & & \multirow[t]{2}{*}{$\begin{array}{l}\text { Sig. } \\
\text { Diff. }\end{array}$} & \multicolumn{2}{|c|}{$\begin{array}{l}\text { Confidence } \\
\text { Interval }\end{array}$} \\
\hline & & & & & & & 0.02 & 0.6 & & $-0,3$ & 0.06 \\
\hline NB2 & 0.7 & 0.4 & $45=$ & 0.7 & 0.5 & 5 & 0.00 & 0 & & 0. & 0.06 \\
\hline NB3 & 1.0 & 0.7 & 431 & 0 & 0.4 & 347 & 0.16 & 4. & $\checkmark$ & 0.09 & 0.22 \\
\hline NB4 & 0.1 & 0.4 & 588 & 0.1 & 0.4 & 459 & 0.06 & 2.4 & $\checkmark$ & 0.02 & 0.10 \\
\hline $\mathrm{S}$ & 0.5 & & 390 & 0.8 & 0.5 & 4 & 0.32 & 9.0 & $\checkmark$ & 0.26 & 8 \\
\hline SB2 & 0. & 1.3 & 214 & 1.0 & 0.6 & 235 & 0.65 & 6.8 & $\checkmark$ & .50 & 0.81 \\
\hline SB3 & 1.0 & 0.5 & 32 & 1.3 & 0.5 & 242 & 0.33 & 7.5 & $\checkmark$ & 026 & 0.40 \\
\hline $\mathrm{SE}$ & 0.4 & 0 & & 0.4 & 0.4 & 3 & 0.03 & 0.9 & & -0.02 & 0.07 \\
\hline EB1 & 0 & 0 & 9 & 6 & 0.4 & 9 & 0.07 & 2.9 & $\checkmark$ & 3 & 0.11 \\
\hline EB2 & 1. & 0.5 & 406 & 1.2 & 1.1 & 375 & 0.09 & 1.6 & & -0.01 & 0.19 \\
\hline EB3 & 1.2 & 0 & 391 & 1.2 & 0.6 & 374 & 0.00 & 0 & & -0.06 & 0.06 \\
\hline EB4 & 0. & 0.3 & 536 & 0.2 & 0.6 & 476 & 0.10 & 3.3 & $\checkmark$ & 0.05 & 0.15 \\
\hline WB1 & 0.3 & 0.6 & 254 & 0.4 & 0.3 & 240 & 0.06 & 1.5 & & -0.01 & 0.14 \\
\hline WB2 & 0. & 0 & 147 & 4 & 0.4 & 214 & 0.15 & 1.8 & & 1 & 0.28 \\
\hline WB3 & 1.1 & 1.0 & 190 & 0.7 & 0.3 & 216 & 0.40 & 5.4 & $\checkmark$ & 0.28 & 0.53 \\
\hline WB4 & 0.1 & 0.5 & 263 & -0.1 & 0.7 & 250 & 0.15 & 2.8 & $\checkmark$ & 0.06 & 0.24 \\
\hline
\end{tabular}




\section{CHAPTER 6. CONCLUSIONS AND RECOMMENDATIONS}

The mean of video detection activations shifted towards earlier activation during night periods by up to 2.01 seconds due to headlight effect. This shift is significant because of the profound difference in measured flowrate with small changes in headway as was demonstrated in Figure 3-1a \& b. For example, if the true headway was 4.0 seconds, yet due to headlight effect and early detector activations the headway was measured as 2.0 seconds, the corresponding measured flowrate would be $1800 \mathrm{VPH}$ instead of the actual value of $900 \mathrm{VPH}$.

To maintain consistency in detector performance under different lighting conditions, gap times must be adjusted by time of day and day of year. This is particularly important in areas of the country where the morning or evening peak periods, occur under low light conditions. These periods may be particularly important as the peak periods are when the most efficient signal operations are desired. However, adjusting gap times by time-of-day and day-of-year may not be feasible to many agencies, especially since the extent of early activation will vary by each signal location due to factors including camera placement, ambient lighting conditions and the degree to which the roadway surface reflects headlights. For example, the headlight effect may be even more pronounced during wet pavement conditions from rainy weather or melting snow, and could not be accounted for in a pre-programmed operation.

The above stop-bar video detection (Cam4 - West Lafayette) provides the smallest variation between night and day operation. The orientation of the camera on the near side of the intersection provides the best position to guard against reflections from the pavement causing false early detections. 
Variations in video detector de-activation times are relatively small between day and night conditions.

\subsection{Recommendations}

The nearside above stop-bar video detection location should be used when possible if more consistent video detection zones are desired. Even with this near side mounting of video detectors, traffic engineers should deploy video detection only when they are willing to accept the stochastic variation in detection zone performance that this paper has quantified at a well lit intersection configured by a highly trained manufacturer representative. Traffic engineers should also be cautioned that the stochastic variation in detection zones would be larger when used at poorly lit intersections.

In the long term, it is essential that video detection manufacturers develop strategies to account for headlight effect in their detection algorithms to provide more consistent video detection zones across different lighting conditions. 


\section{LIST OF REFERENCES}

1. Rhodes A., D. Bullock, J. Sturdevant, Z. Clark, and D. Candey. Evaluation of Stop Bar Video Detection Accuracy at Signalized Intersections. Transportation Research Board (\#05-0384), TRB, National Research Council, Washington, D.C. 2005.

2. Grenard, J., D. Bullock, and A. P. Tarko. Evaluation of Selected Video Detection Systems at Signalized Intersections. Publication

FHWA/IN/JTRP-2001/22. Joint Transportation Research Program, Indiana Department of Transportation, Purdue University, 2001.

3. MacCarley, C. A., and J. Palen. Evaluation of Video Traffic Sensors for Intersection Signal Actuation: Methods and Metrics. Transportation Research Board, National Research Council, Washington, D.C., 2003.

4. Bonneson, J., and M. Abbas. Video Detection for Intersection and Interchange Control. Publication FHWA/TX-03/4285-1. Texas Transportation Institute, Texas Department of Transportation, FHWA, 2002.

5. Baculinao, N. M. Video Detection Technology - Is it Ready to Replace Loops? Compendium of Papers for the 1998 ITE District 6 Meeting in San Jose, CA, Institute of Transportation Engineers, Washington, D.C., 1998.

6. Ginsberg, S. "Traffic-Light Timing System Only Leaves Drivers Idling: Vaunted Cameras Along Route 7 on the Blink." Washington Post, November 6, 2005, natl. ed. : Page C04. 